\title{
The Elucidation of the Ground State in the H-Atom-Model of Niels Bohr and its Application on the Bond-Length Computation in the $\mathrm{H}_{2}$-Molecule
}

\author{
Thomas Allmendinger* \\ Independent Scholar, CH-8152 Glattbrugg/Zürich, Switzerland
}

\begin{abstract}
Received: January 23, 2018; Accepted: February 15, 2018; Published: February 20, 2018
*Corresponding author: Dr. Thomas Allmendinger, Zunstrasse 1, CH-8152 Glattbrugg/Zürich, Switzerland. Tel/Fax: +41448101733; Email: inventor@sunrise.ch
\end{abstract}

\begin{abstract}
The phenomenon of spin-orbit-coupling let suppose that the electron spin, which exhibits the character of an oscillator but which cannot be explained by classical physical laws, is herewith identified to be responsible for a minimal angular momentum - given by Planck's constant - which determines the ground state of a single $\mathrm{H}$-atom according to Bohr's model. Thereby, the frequencies of the spin and of the revolution are identically-equal which leads to a standing wave. However, in contrast to the theory of Classical Quantum Mechanics where the electron-orbital is spherical even in the ground state, here the electron trajectory is planar.
\end{abstract}

Applying the same concept for the $\mathrm{H}_{2}$-molecule, i.e. assuming the maintenance of the basic angular momentum for both $\mathrm{H}$-atoms at the $\mathrm{H}-\mathrm{H}$ bond, a comparatively simple computation method for the intrinsic variables could be found regarding several force equilibria between electrons and protons. However, the relation could not be formulated generally but had to be adapted to four conceivable arrays. Since in any case a variety of combinations of the relevant parameters exists fulfilling the given condition, the total energy has to be introduced for determining the preferred stable state which is given by an energy minimum. As the computations yielded, only the so-called intranuclear-sympodial array enables a stable state being given at a bond length of $0.746 \AA$, and hence matching perfectly the desired value determined by XRD-crystallography. This result exceeds the accuracy of the next best result which was obtained by the Classical Quantum Mechanics, namely $0.71 \AA$.

However, the difference between the resulting bond energies for the two methods is enormous. If these values are compared with the experimentally determined literature value, the traditional method appears to be accurate. However, as consideration of plausibility yielded, this literature value is probably not true but significantly too high, so it cannot be adduced to stringent evidence. If the here proposed approach is correct, implicating well-defined electron trajectories, the theory of Classical Quantum Mechanics, as well as Heisenberg's uncertainty principle, cannot be maintained since it is not possible that the reality can be described in terms of two different theories.

Keywords: $\mathrm{H}$-atom-model; Electron-spin; $\mathrm{H}_{2}$-molecule; Bondlength computation; Bond-strength;

\section{Introduction}

In 1913, shortly before the beginning of the First World War, the Dane Niels Bohr published an article entitled "On the Constitution of Atoms and Molecules", focussing the H-atom [1]. Starting from Rutherford's atom model, which assumed a heavy positively charged nucleus in the centre and a light electronic envelope, and applying Planck's quantum theory of radiation, introduced in 1900 [2] and supplemented by Albert Einstein in 1905 [3], he suggested that - as a result of (UV-) radiative excitation - well-defined orbits were occupied by the electron in the H-atom. His model could widely explain the line-spectrum of hydrogen which had been observed astronomically in certain Space regions as well as in terrestrial experiments using low pressure tubes, and wherein remarkable regularities had been originally found by Balmer [4], later revealed by Rydberg. The essential point of his consideration may be expressed by the following statement made in: [1] In any molecular system consisting of positive nuclei and electrons in which the nuclei are at rest relative to each other and the electrons more in circular orbits, the angular momentum of every electron round the centre of its orbit will in the permanent state of the system be equal to $h / 2 \pi$, where $h$ is Planck's constant." (Therein, «permanent state» means «ground state» of the electron). Moreover, he found that the angular momenta of the excited states are integral multiples of the angular momentum of the ground state. It is worth mentioning that Planck's constant $h$, which he himself denoted as «elementares Wirkungsquantum» («elemental action quantum»), represents the product of energy and time (delivering the dimension $\mathrm{J} \times \mathrm{s}$ ), being equal to the dimension of an angular momentum. Hence Bohr's atom model may be considered as the outset of modern quantum mechanics.

However, this fundamental work comprises some capital errors in [1] which nobody has been aware so far. This led the scientists to go in a wrong direction, finally leading to the orbital theory which is still considered as commonly agreed and hence designed as «Classical Quantum Mechanics», in spite of its unintelligibility.

Right at the start, it has to be mentioned that the dimensions 
of the numerical values were usually omitted. That makes more difficult to understand them and to verify the mathematical relations, but this is not crucial since the delivered numerical results are correct.

Rather, Bohr's cardinal error was initiated by the logical incompatibility of two different expressions describing the relation between the energy $W$ and the frequency of revolution $\omega$ : on the one hand he used the - principally correct - relation

$$
\omega=\frac{\sqrt{2}}{\pi} \frac{W^{3 / 2}}{e \cdot E \sqrt{m}}
$$

( $e=$ charge of the electron, $E=$ charge of the proton, $m=$ electron mass)

while, on the other hand, he inserted the quantum relation

$$
W=\tau \cdot h \cdot \frac{\omega}{2} \rightarrow \omega=\frac{2 W}{\tau \cdot h}
$$

\section{( $\tau$ = quantum number, $h=$ Planck's constant $)$}

But these two expressions cannot be simultaneously valid since they imply a different power of relation with respect to $W$. This error results from Bohr's assumption that the electron revolution around the nucleus is identically equal to the oscillation which is assumed in Planck's theory. This may be refuted by the fact that, within an oscillation, potential energy is perpetually converted into kinetic energy, and vice versa, while, during a revolution, the kinetic energy is constant. As a consequence, in addition to the revolution, an oscillation of the electron should be supposed, which is responsible for the interaction with light.

This perception coincides with a hypothesis which was made in 1924 by Louis De Broglie in his thesis, assuming a wavy electron motion, and leading to the term «wave mechanics». It not only explained the deflection of electron beams on thin metal foils (cf. for instance [5]) but also - and in particular - the occurrence of well-defined electron trajectories in the excited states as a result of standing waves. However, a vivid $\mathrm{H}$-atom model comprising such an electron motion could not be found at that time. Instead, Classical Quantum Mechanics gained acceptance actually representing insofar a statistical theory as it assumes probabilities of electron presence in orbitals instead of well-defined electron trajectories, and disregarding Bohr's angular momentum theorem. It may be interesting to notice that this theory was established between the two World Wars, to a considerable extent by physicists such as Schrödinger, Heitler and Pauli, working in Zürich (Switzerland) which promised more security in this unstable political situation. Conceivably, the unstable situation became also apparent in the physical theory of that time, being distinguished by the uncertainty principle of Werner Heisenberg.

As could be demonstrated within the author's recent publication, it is indeed feasible to mathematically describe such a vivid model exhibiting precisely describable three-dimensional oscillating electron trajectories in the excited states, while the orbit in the ground state is planar [6]. This planarity of the ground state correlates to Bohr's model but contradicts the Classical Quantum Mechanics which assumes a spherical ground state, represented by the 1s-orbital. When chemical bonds are focussed, as it is here the case, solely the ground states of the atoms are relevant, while excited states are primarily relevant for AAS-spectroscopy.

In 1925, an additional aspect was delivered by Goodsmith and Uhlenbeck, concerning the spectra recorded in the presence of a magnetic field [7, 8, 9]. As Zeeman had observed and published already in 1887, such fields induced shifts and widenings of spectral lines [10]. Moreover, in some cases the multiplicity of certain lines turned out to be larger than expected, that phenomenon being denoted as «anomalous Zeeman-effect» [11]. For its interpretation, Goudsmith and Uhlenbeck suggested that the electron exhibits an own intrinsic angular momentum being incident to a magnetic momentum which subsequently was denoted as «spin». It was afterwards implemented into the usual theory by Pauli and by Dirac, leading to the well-known «Pauli Principle». However, nobody bothered about its relevance to the Bohr model since it was shelved forever.

Moreover, the main interest was focussed on the excited states of the electrons - thus interpreting spectroscopic phenomena -, even if the orbital concept was applied on the ground states, too, delivering one spherical s-orbital, three aligned p-orbitals, and further aligned orbitals. Thereby, it appears odd that, for explaining the «Aufbau-Principle» of the Periodic System, as well as - particularly - the fourfold tetrahedral valence of atoms, the orbitals must be «hybridized» since the p-orbitals are orthogonal. However, this hybridization means solely a formalistic act, being not really applicable in chemical practice by exact computations. Rather, such a computation would have to be initially applied to the simplest case which is represented by the $\mathrm{H}-\mathrm{H}$ bond in the hydrogen molecule.

While Bohr did not deliver an explanation of chemical bonds - contrary to the notification in the title of his publication [1] -, Heitler and London presented in 1927 a method for expressing the covalent bond in the $\mathrm{H}_{2}$-molecule, applying the orbital theory but not describing the computation in detail, delivering distance/ energy-curves as shown in Figure 1, and assuming that the true bond lengths are reached when the total energy is minimal due to its most stable state [12]. The energy values in the published curves are usually normalized by reference to the energy content of two separate $\mathrm{H}$-atoms which cannot be directly measured but calculated according to Bohr's model, and verified by the accordance with spectral data (see for example [6]; its value is $-4.3593 \times 10^{-18} \mathrm{~J} /$ molecule $=-27.2 \mathrm{eV} /$ molecule $)$. Heitler cited it in his later published book about elemental wave mechanics [13], and the fact that this method has been alleged in the modern textbook of Haken and Wolf let suppose that it is still valid. Since it is possible to calculate the bond-length in the $\mathrm{H}_{2}$-molecule, it is possible to verify the method by comparing its result with the value of the bond length which has been exactly determined by an alternative method, namely by the crystallographic one using X-rays [14]. The relevant data may be found in the familiar 'Handbook of Chemistry and Physics' (55 ${ }^{\text {th }}$ Ed.; reproduced by 


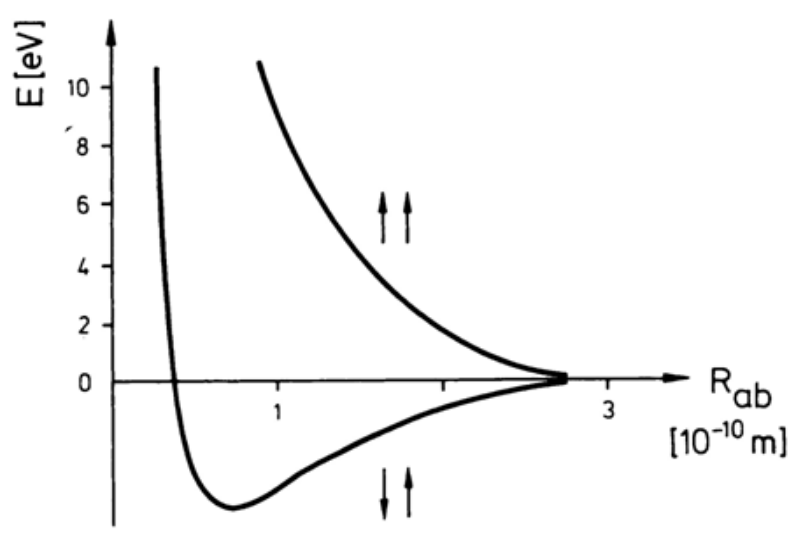

Figure 1: Computed distance-energy diagram for the H-H bond, regarding two different spin-orientations, according to [14].

permission from International Tables for X-ray Crystallography), amounting to $0.74611 \times 10^{-10} \mathrm{~m}(=0.74611 \AA)$.

The experimental evaluation of the bond energy - or inversely of the dissociation energy - is much more delicate since it involves thermodynamic methods and theory needing adequate equipment. An early approach was made by Bichowsky and Copeland within the scope of a thesis using a caloric method based on the effusion theory of Knudsen $[15,16]$. The method is not readily understandable - and examinable - since complex steady state dynamic gas flow conditions are applied where tubes and capillaries with different diameters are used. Thereby, in a first step partly-dissociated hydrogen gas is generated by expansion from high to low pressure, due to Ostwald's dissipation law, while in a second step the volume contraction as well as the temperature change - due to the catalytically enabled recombination of $\mathrm{H}$-atoms to $\mathrm{H}_{2}$-molecules- is measured by a calorimeter. The percentage of atomic hydrogen causing the change is determined by the pressure between the different states. With respect to the relatively high average dissociation portion of $26.6 \%$ for $\mathrm{H}_{2}$ into $\mathrm{H}$-atoms, the high average energy value of $-105.3 \mathrm{kcal} / \mathrm{mole}=-440.7 \mathrm{~kJ} /$ mole does not at all appear plausible: the usual thermodynamic relation between Gibbs free energy $\Delta \mathrm{G}$ (in kcal) and the equilibrium constant $K$, described by the equation

$$
\Delta G=\Delta H-T \Delta S=-R T \ln K \rightarrow \ln K=-\frac{\Delta G}{R T}
$$

whereby $R T=0.592 \mathrm{kcal} / \mathrm{mole}$ when $T=298 \mathrm{~K}$

would let suppose a very high equilibrium constant since the value of $\Delta G$ does not considerably differ from the value of $\Delta H$. On the other hand, the existence of dissociated H-atoms at low pressures is attested by the feasibility of atomic hydrogen spectra which deliver the empiric foundations for Bohr's atom model.

Later, measurements of bond strengths were made using mass-spectrometry for determining the particle concentrations in the gas-phase. The strength of a chemical bond is defined as the heat of the dissociation reaction. As mentioned in the explanatory notes of the 'Handbook of Chemistry and Physics', bond strengths are not equal to - and may differ considerably from - mean bond energies derived solely from thermochemical data on molecules and atoms. It should be realized that the total energy consists of the components kinetic translation energy (being correlated to the sensible heat), the molecular rotation energy, and the internal vibration energy of the molecules within the bonds.

In most cases, sublimation vapours over solid bodies were investigated [17]. Unfortunately, the quotation [18], which is alleged in the 'Handbook' for the $\mathrm{H}_{2}$-case, is not correct since it concerns gaseous metal nitrides, while the correct citation could not be found so far. Thus solely the therein cited value of -104.2 $\mathrm{kcal} / \mathrm{mole}=-436.2 \mathrm{~kJ} /$ mole can be noticed here. It is very close to the above cited value of Bichowsky and Copeland, but it should be queried likewise.

In addition, the following comparison may be made using the literature values for related bond strengths given in the 'Handbook': The strength of the $\mathrm{H}-\mathrm{Cl}$ bond is $-431.6 \mathrm{~kJ} / \mathrm{mole}$, while the one of the $\mathrm{Cl}-\mathrm{Cl}$ bond is $-243.1 \mathrm{~kJ} / \mathrm{mole}$. First of all, it sounds odd that the highly polar and thus strong $\mathrm{H}-\mathrm{Cl}$ bond would be even weaker than the $\mathrm{H}-\mathrm{H}$ bond when the above mentioned value $-436.2 \mathrm{~kJ} / \mathrm{mole}$ is assumed. Moreover, if the energy-theorem of Hess is regarded, the bond energy (corresponding to the bond strength) of the $\mathrm{H}-\mathrm{Cl}$ bond should be the sum of the bond energies of the $\mathrm{Cl}-\mathrm{Cl}$ and the $\mathrm{H}-\mathrm{H}$ bond. This yields, if the value for the $\mathrm{Cl}-\mathrm{Cl}$ bond is accepted as correct, for the $\mathrm{H}-\mathrm{H}$ bond the value of only $188.5 \mathrm{~kJ} /$ mole, which is 2.3 fold smaller than the given value!

Keeping these objections in mind, the results of the abovecited quantum mechanical computations are compiled in Table 1. Obviously, the bond length values are diverse, while none of them matches exactly the effective value of $0.74611 \AA$. The remarkable differences were not explained by the authors. The energy minimum values differ even more. The value given by Haken and Wolf is close to the literature value quoted in the 'Handbook' $(-4.25 \mathrm{eV} /$ molecule $=-410.1 \mathrm{~kJ} /$ mole, compared to $-436.2 \mathrm{~kJ} / \mathrm{mole}$ ) [14]. However, this cannot be considered as a proof because of the above-mentioned objections. Overall, while quantum mechanics usually arrogates highest precision, the here cited empirical evidence for maintaining this theory is indeed quite poor, not least since the results are not consistent.

Table1: Known quantum mechanical computation results for the $\mathrm{H}-\mathrm{H}$ bond

\begin{tabular}{|c|c|c|c|}
\hline \multicolumn{2}{|l|}{ Authors/Quotation } & Bond Length & Energy Minimum \\
\hline \multicolumn{2}{|c|}{ Heitler \& London [12] Fig. 1} & $0.85 \AA$ & $-2.35 \mathrm{eV}$ \\
\hline Heitler [13] & $\begin{array}{l}\text { Bild } 34 \\
\text { Tab. } 9\end{array}$ & $\begin{array}{l}1.00 \AA \\
0.85 \AA\end{array}$ & $\begin{array}{l}-3.35 \mathrm{eV} \\
-3.20 \mathrm{eV}\end{array}$ \\
\hline Haken \& Wolf [14] & Abb. 4.12 & $0.71 \AA$ & $-4.25 \mathrm{eV}$ \\
\hline
\end{tabular}

\section{Description of the Present Alternative Approach}

In contrast, here a calculation of the bond length in the $\mathrm{H}_{2}$ molecule is presented using the approach of the Bohr-model in the ground state, and according to the previous publication [6]. However, an additional assumption concerning the ground state has to be made which has not been explicitly formulated so far. It corresponds to Bohr's preliminarily cited statement, but it can 
be founded by a phenomenon which Bohr had not yet known, namely the spin which represents an intrinsic and not any further explicable quality of the electron, behaving like an oscillator with a magnetic moment. Due to the empirical fact that there exists a so-called spin-orbit coupling, i.e. that the magnetic spin moment of the electron is nearly equal to its magnetic orbit moment, the spin may be assumed as the real cause for the stability of the ground state of the H-atom. Hence the spin may be imagined as an oscillator which acts perpendicularly to a planar electron orbit, inducing a further planar electron orbit which is identically equal to the orbit of the Bohr-model in the ground state. Thereby, the oscillation frequency of the spin must be equal to its revolution frequency. As a consequence, the angular momentum of the electron orbit in the ground state can be regarded as unalterable, not only in the single $\mathrm{H}$-atom, but also in the $\mathrm{H}_{2}$-molecule. This hypothesis is necessary for calculating the bond-length in the molecule where precisely defined electron orbits are assumed, slightly differing from the orbits of the single $\mathrm{H}$-atoms in their ground states.

Hereto it is advisable, in a first step, to visually describe the principal array of the two protons (nuclei) and the two electrons, whilst in a second step numerical computation may reveal the accurate array by matching the effective bond-length, here denoted as $d$ = distance. But $\mathrm{d}$ is not the only variable (besides the electron velocity $u$ ): the deflection $a$ and the radius $r$ (which is not identically equal to the Bohr-radius) is additionally needed for describing the positions of the electrons. The four sterically conceivable arrays are scheduled in Figure 2, indicated according to the positions of the electrons as intranuclear-antipodal, intranuclear-sympodal, extranuclear-antipodal, and extranuclearsympodal.

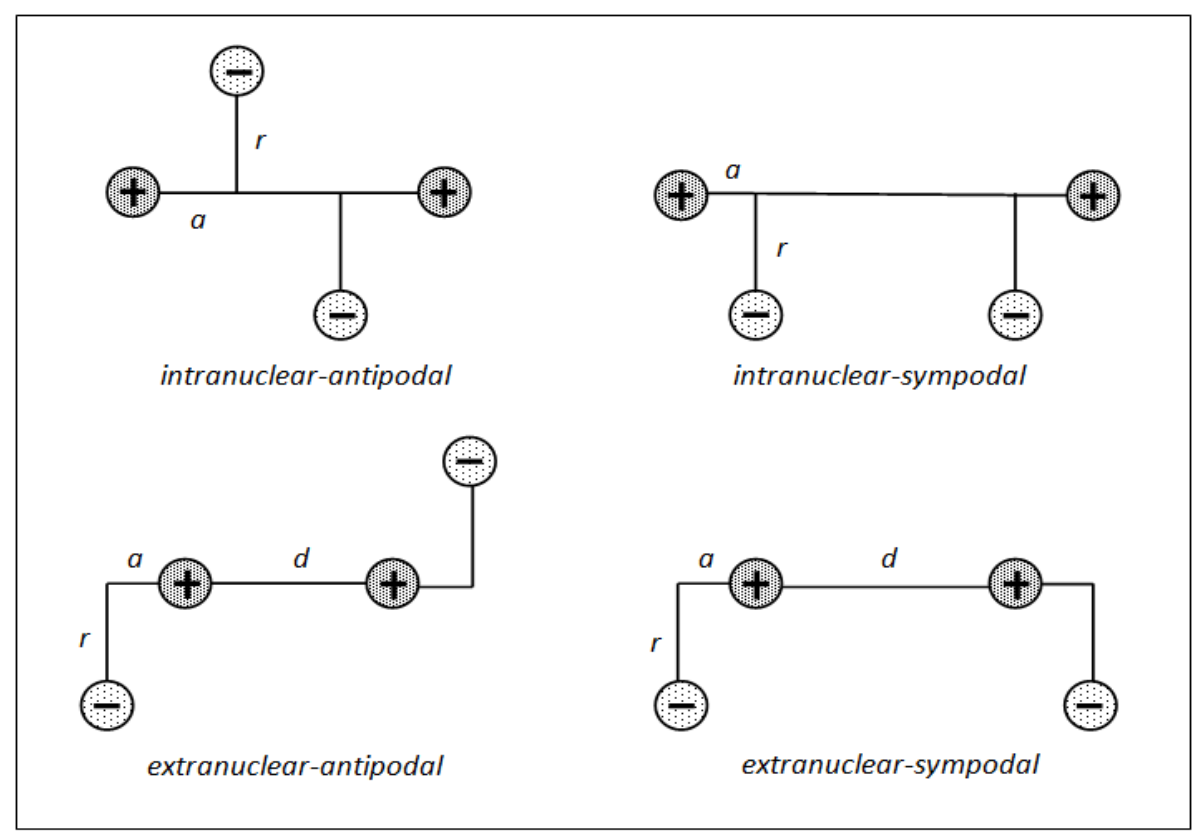

Figure 2: The four possible arrays of the electrons.

The computation has to be based on possible force equilibria using general physical laws, i.e. the Coulomb Law and the relation for the centrifugal force, as well as the quantum condition given by the angular momentum of the electron which is assumed to be invariable and identically equal to the angular momentum of the angular momentum in a single $\mathrm{H}$-atom. Subsequently, the relevant equations have to be combined, delivering an expression for the distance $d$ as a function of the other parameters. This means that a variety of solutions fulfilling this condition exists. Thus any combination of arbitrarily assumed variables $d$ and $a$ determines a distinct value of $r$, but these $r$ values can only be evaluated by nested intervals.

Finally, the total energies of the molecular system have to be computed as a function of several $d / a / r$ - combinations, whereby the $d$ value, which is accompanied by the energy minimum, should match the desired $d$ value, while the total energy should become equal to the sum of the energies of the two separated H-atoms in the ground state when $\mathrm{d}$ becomes infinite.

\section{Computation of the Intranuclear-Antipodal Array}

Since the computation of such an array needs some particular knowledge which is not a priori intelligible, an example is given here in detail, affecting the intranuclear-antipodal array. This case is not only the most difficult one for computation but also the most prominent one representing the accurate array. The intranuclear-sympodal array and the extranuclear-antipodal array turned out to imply no energy minima, thus they remain out of consideration. The extranuclear-sympodal array implicates energy minima, but they are more positive than those of the two single $\mathrm{H}$-atoms in the ground state. Nevertheless, the respective computation, resulting in a distance/energy-diagram, will be demonstrated in the next chapter. 
As mentioned in the subtitle of the respective Figure 3, preferably the auxiliary terms $b, R_{1}$ and $R_{2}$ are introduced which facilitates formulating. They are defined by the equations

$$
\begin{aligned}
& b=\sqrt{(d-2 a)^{2}+4 r^{2}} \\
& R_{1}=\sqrt{a^{2}+r^{2}} \\
& R_{2}=\sqrt{(d-a)^{2}+r^{2}}
\end{aligned}
$$

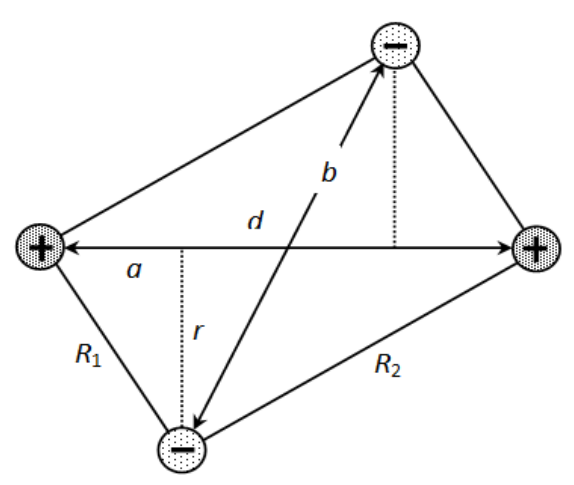

Figure 3: Intranuclear-antipodal array, with the auxiliary terms $b, R_{1}$ and $R_{2}$.

Moreover, it is noteworthy that in Fig. 3 the repelling Coulomb-forces are indicated by double-arrows, while the attracting Coulomb-forces are indicated by simple lines and the rotation radius of the electrons by a dashed line. The Coulomb Law is simplified by using the constant $\mathrm{K}$ which comprises the induction constant $\varepsilon_{0}$ according to the relation

$$
K=\frac{e^{2}}{4 \pi \varepsilon_{0}}
$$

( $e=$ elementary charge, being quantitatively equal for proton and electron).

A first difficulty arises from the inclinations of some Coulomb-forces inducing partial forces. They can be expressed by multiplying the primary Coulomb-force by the ratios of the lengths belonging to the partial Coulomb-forces. For instance, the horizontal component referring to a of the Coulomb-force between the nucleus and the closer electron referring to $R_{1}$ is given by the relation $\frac{K \cdot a}{R_{1}^{3}}$, while the vertical component referring to $\mathrm{r}$ amounts to $\frac{K \cdot r}{R_{1}^{3}}$.

A further difficulty arises from the question how the force equilibria, particularly the horizontal ones, must be focussed, and how the algebraic signs must be defined with respect to attraction and repulsion. Thereby it turned out that, for formulating a certain force equilibrium, one particle has to be focussed relative to which the forces of the other three particles has to be formulated, while the direction of attracting forces may be signed arbitrary but contrary to the direction of repelling forces. For instance: The left proton in Fig. 3 is repelled by the right proton, but attracted by both electrons in the opposite direction. The same is true for the right proton but vice versa. However, the left electron is repelled by the right electron and attracted by the left proton in the same direction, but pulled by the right proton in the contrary direction. Hence, the following two equations can be established:

For the proton: $\frac{K}{d^{2}}=\frac{K \cdot a}{R_{1}^{3}}+\frac{K(d-a)}{R_{2}^{3}}$

For the electron: $\frac{K(d-a)}{R_{2}^{3}}=\frac{K \cdot a}{R_{1}^{3}}+\frac{K(d-2 a)}{2 b^{3}}$

Considering the vertical force equilibrium, first of all - and easily - the Coulomb-forces between the two nuclei (protons) and one electron can be taken into account as attracting forces, while the centrifugal force of the rotating electron, related to the connecting line of the two nuclei, can be assumed as diametrically opposite force. However, the repellent Coulomb force of the other electron has to be additionally regarded. Thereby the question arises, which distance has to be applied: the total distance between the two electrons, i.e. $2 r$, or only the distance between the connecting line of the two nuclei and the electron, i.e. $1 r$ ? As the subsequent comparative computation yielded, the former variant is accurate. Thus the respective force equilibrium equation must be written

$$
\frac{K \cdot r}{R_{1}^{3}}+\frac{K \cdot r}{R_{2}^{3}}=\frac{m \cdot u^{2}}{r}+\frac{K \cdot r}{b^{3}}
$$

( $m=$ electron mass, and $u=$ electron velocity).

Finally, the quantum condition can be formulated for each electron, yielding equation (7):

$$
m \cdot u \cdot r=\frac{h}{2 \pi} \rightarrow u=\frac{h}{2 \pi m \cdot r} \rightarrow m \cdot u^{2}=\frac{h^{2}}{4 \pi^{2} m \cdot r^{2}}
$$

Now the equations (4), (5), (6) and (7) can be combined, delivering equation (8):

$$
\frac{1}{d^{2}}=2(d-a)\left(\frac{C}{r^{4}}-\frac{1}{R_{1}^{3}}\right)+\frac{1.5 d-a}{b^{3}}
$$

with $C=\frac{h^{2}}{4 \pi^{2} m \cdot K}=0.5292 \times 10^{-10} \mathrm{~m}$ (or $\AA$ ).

Using equation (8), it is possible to find by nested intervals for any combination of arbitrarily assumed variables $d$ and $a$ a distinct value of $r$, preferably by means of 'Excel' (Microsoft). Thereby, the distance $d$ should be the primary variable while the deflection $\mathrm{b}$ is the secondary variable. Thus for each predetermined $d$-value variable $b$-values may be supposed. By varying $r$, the value of $1 / d^{2}$, computed according to equation (8), must be on par with the value of $1 / d^{2}$ being computed on bases of the predetermined value of $d$. This can easily been ascertained by considering the quotient of the two values which must exactly be 1 .

However, for limiting the infinite number of such combinations, a further condition is needed. It is given by the principle of minimal total energy which is of eminent importance in chemistry. The total energy of the hydrogen molecule, consisting of the total potential and the total kinetic energy of all particles in the molecule, can be expressed by formula (9):

$$
E_{t o t}=K\left(\frac{1}{d}+\frac{1}{b}-\frac{2}{R_{1}}-\frac{2}{R_{2}}\right)+\frac{D}{r^{2}}
$$


with $K=\frac{e^{2}}{4 \pi \varepsilon_{0}}=2.307 \times 10^{-28} \mathrm{~J} \times \mathrm{m}$ and $D=\frac{h^{2}}{4 \pi^{2} m}=1.2208510^{-38}$ $\mathrm{J}^{2} \mathrm{~s}^{2} \mathrm{~kg}^{-1}$

Moreover, it is advantageous to start with the real, empirically determined d-value, while similar computations may follow supposing adjacent d-values. The results of such a computation are exemplarily displayed in Table 2 where A corresponds to the formula [8]. Therein, the minimal energy (being characterized by a maximal negative value) is emphasized by shading. It is fulfilled when $d=0.7461 \AA$, $a=0.115 \AA$, and $r=0.47249 \AA$.

Analogously, the energy minima were computed for several further distances, namely from 0.55 till $2.0 \AA$ A. The plot of the total minimal energy as a function of the distance $d$ is displayed in Figure 4. Its minimum at $-4.6375 \times 10^{-18} \mathrm{~J}$ matches exactly the desired $d$-value of $0.75611 \AA$, while it appears tending to the total energy of two $\mathrm{H}$-atoms $\left(-4.3593 \times 10^{-18} \mathrm{~J}\right)$ at high distances. Therefore, according to this consideration, the resulting stabilization energy (usually called «exchange energy») equates to the difference of these two energies, namely $-0.2782 \times 10^{-18}$ J, amounting to approx. $6.4 \%$. (A comparison to the literature values is made in the last chapter). Based on these values, an exact model of the $\mathrm{H}_{2}$-molecule can be drafted (Figure 5 and Table $3)$. As expected, the rotation radius in the $\mathrm{H}_{2}$-molecule is slightly $(10.8 \%)$ smaller than the Bohr-radius in a single H-atom, namely $0.472 \AA$ instead of $0.529 \AA$.

Table 2: Excel compilation to the nested intervals computation of the intranuclear-antipodal- array for a given value of $d$ (unit for lengths: $\AA=10^{-10}$ m; energy unit: $10^{-18} \mathrm{~J} ; \mathrm{C}=0.5292 \AA$ )

\begin{tabular}{|c|c|c|c|c|c|c|}
\hline$d$ & $a$ & $r$ & $\mathrm{~A}=\underset{(1.5 d-a) / b^{3}}{(d-2 a) \cdot\left(\mathrm{C} / r^{4}-1 / R_{1}^{3}\right)+}$ & $\mathrm{B}=1 / d^{2}$ & B/A & $E_{\text {tot }}$ \\
\hline 0.74611 & 0.00 & 0.461035 & 1.79638 & 1.79636 & 1.0000 & -4.4879 \\
\hline 0.74611 & 0.005 & 0.460825 & 1.79633 & 1.79636 & 1.0000 & -4.5021 \\
\hline 0.74611 & 0.01 & 0.460700 & 1.79639 & 1.79636 & 1.0000 & -4.5154 \\
\hline 0.74611 & 0.02 & 0.460710 & 1.79645 & 1.79636 & 1.0000 & -4.5399 \\
\hline 0.74611 & 0.05 & 0.462600 & 1.79635 & 1.79636 & 1.0000 & -4.5954 \\
\hline 0.74611 & 0.06 & 0.463740 & 1.79645 & 1.79636 & 1.0000 & -4.6083 \\
\hline 0.74611 & 0.07 & 0.465085 & 1.79635 & 1.79636 & 1.0000 & -4.6187 \\
\hline 0.74611 & 0.08 & 0.466580 & 1.79643 & 1.79641 & 1.0000 & -4.6266 \\
\hline 0.74611 & 0.09 & 0.468200 & 1.79635 & 1.79636 & 1.0000 & -4.6322 \\
\hline 0.74611 & 0.10 & 0.469890 & 1.79642 & 1.79636 & 1.0000 & -4.6358 \\
\hline 0.74611 & 0.11 & 0.471620 & 1.79642 & 1.79636 & 1.0000 & -4.6374 \\
\hline 0.74611 & 0.115 & 0.472490 & 1.79634 & 1.79636 & 1.0000 & -4.6375 \\
\hline 0.74611 & 0.12 & 0.473350 & 1.79638 & 1.79636 & 1.0000 & -4.6372 \\
\hline 0.74611 & 0.13 & 0.475040 & 1.79634 & 1.79636 & 1.0000 & -4.6353 \\
\hline 0.74611 & 0.14 & 0.476650 & 1.79641 & 1.79636 & 1.0000 & -4.6319 \\
\hline 0.74611 & 0.15 & 0.478155 & 1.79635 & 1.79636 & 1.0000 & -4.6269 \\
\hline 0.74611 & 0.16 & 0.479510 & 1.79642 & 1.79636 & 1.0000 & -4.6205 \\
\hline 0.74611 & 0.17 & 0.480690 & 1.79645 & 1.79636 & 1.0000 & -4.6127 \\
\hline 0.74611 & 0.20 & 0.482860 & 1.79635 & 1.79636 & 1.0000 & -4.5807 \\
\hline 0.74611 & 0.25 & 0.479860 & 1.79639 & 1.79636 & 1.0000 & -4.4899 \\
\hline 0.74611 & 0.35 & 0.419840 & 1.79637 & 1.79636 & 1.0000 & -3.6734 \\
\hline
\end{tabular}

\section{The Extranuclear-Sympodal Array}

According to respective computations which are not discussed here in detail, the intranuclear-sympodal array as well as the extranuclear-antipodal array turned out to imply no energy minima for any distance, thus they remain out of consideration. The extranuclear-sympodal array implicates energy minima, but they are more positive than those of the two separated $\mathrm{H}$-atoms in the ground state. Nevertheless the respective computation is demonstrated here.

The respective array is scheduled in Figure 2. Additionally and analogously to the previous case, the distance b between the two electrons is introduced. The basic equations can be established using the same rules as given above, which leads to the following system: 


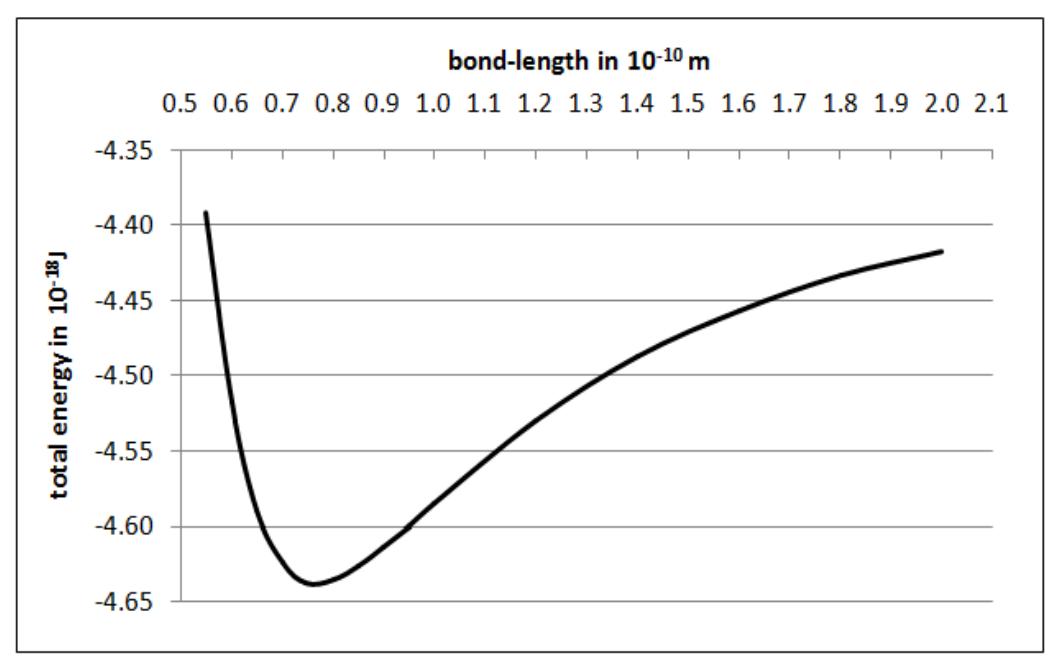

Figure 4: Total energy as a function of the bond-length (= distance $d$ ) at the intranuclear-antipodal array of the $\mathrm{H}_{2}$-molecule.

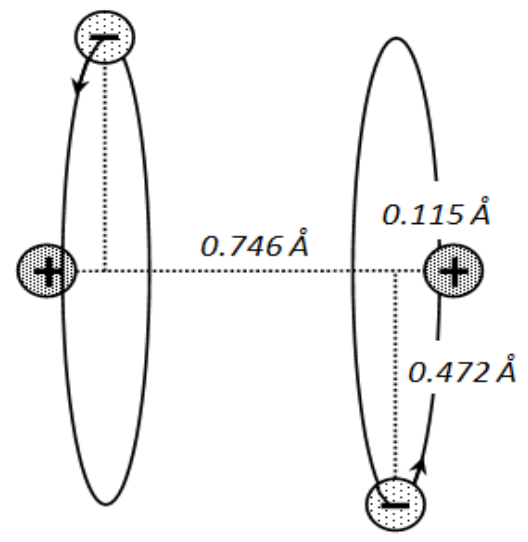

Figure 5: Model of the $\mathrm{H}_{2}$-molecule (true to scale).

Table3: Basic data to Figure 4 and 5

\begin{tabular}{|c|c|c|c|c|}
\hline $\boldsymbol{d}$ & $\mathbf{E}_{\text {tot }}$ & $\boldsymbol{a}[\AA]$ & $\boldsymbol{r}[\AA]$ & $\boldsymbol{a} / \boldsymbol{d}$ \\
\hline 0.55 & -4.392 & 0.100 & 0.401 & 0.182 \\
\hline 0.60 & -4.518 & 0.110 & 0.421 & 0.183 \\
\hline 0.65 & -4.590 & 0.120 & 0.441 & 0.185 \\
\hline 0.70 & -4.625 & 0.115 & 0.458 & 0.164 \\
\hline 0.746 & -4.638 & 0.115 & 0.472 & 0.154 \\
\hline 0.80 & -4.636 & 0.105 & 0.485 & 0.131 \\
\hline 0.85 & -4.627 & 0.095 & 0.492 & 0.112 \\
\hline 0.90 & -4.614 & 0.085 & 0.500 & 0.094 \\
\hline 0.95 & -4.600 & 0.075 & 0.506 & 0.079 \\
\hline 1.00 & -4.585 & 0.065 & 0.511 & 0.065 \\
\hline 1.20 & -4.530 & 0.040 & 0.521 & 0.033 \\
\hline 1.40 & -4.487 & 0.025 & 0.526 & 0.018 \\
\hline 1.60 & -4.457 & 0.020 & 0.529 & 0.013 \\
\hline 1.80 & -4.434 & 0.008 & 0.529 & 0.004 \\
\hline 2.00 & -4.418 & 0.006 & 0.530 & 0.003 \\
\hline
\end{tabular}

Modified geometric relations:

$$
\begin{gathered}
b=d+2 a \\
R_{1}=\sqrt{a^{2}+r^{2}} \\
R_{2}=\sqrt{(d+a)^{2}+r^{2}}
\end{gathered}
$$

Horizontal force equilibrium for the proton:

$$
\frac{K}{d^{2}}+\frac{K \cdot a}{R_{1}^{3}}=\frac{K(d+a)}{R_{2}^{3}}
$$

Horizontal force equilibrium for the electron:

$$
\frac{K}{b^{2}}=\frac{K \cdot a}{R_{1}^{3}}+\frac{K(d+a)}{R_{2}^{3}}
$$

Vertical force equilibrium:

$$
\frac{K \cdot r}{R_{1}^{3}}+\frac{K \cdot r}{R_{2}^{3}}=\frac{m \cdot u^{2}}{r}
$$

The quantum relation is unchanged [7]. Hence the combination of these equations yields

$$
\frac{1}{d^{2}}=2(d+a)\left(\frac{C}{r^{4}}-\frac{1}{R_{1}^{3}}\right)-\frac{1}{b^{2}}
$$

Since formula for the total energy is unchanged, too, the bond-lengths $d$ with minimal energies may be found by nested intervals, delivering the diagram in Figure 6 and Table 4 [9]. It reveals that that all energy values are higher than the sum of two single $\mathrm{H}$-atoms being $-4.3593 \times 10^{-18} \mathrm{~J}$, which means that this array does not allow a stable bonding state.

\section{Summary, Discussion and Conclusions}

The phenomenon of spin-orbit-coupling let suppose that the electron spin, which exhibits the character of an oscillator but which cannot be explained by classical physical laws, is herewith identified to be responsible for a minimal angular momentum given by Planck's constant - which determines the ground state of 


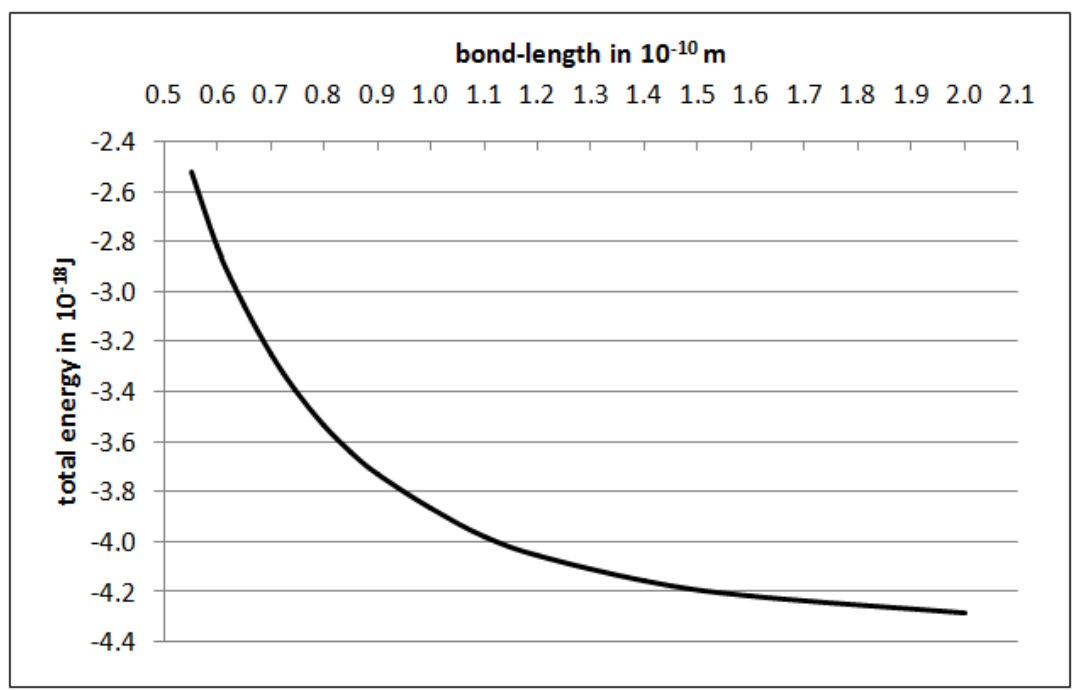

Figure 6: Total energy as a function of the bond-length (= distance d) at the extranuclear-sympodal array of the $\mathrm{H}_{2}$-molecule.

\begin{tabular}{|c|c|c|c|c|}
\hline \multicolumn{6}{|c|}{ Table 4: Basic data to Figure 6} \\
\hline $\boldsymbol{d}$ & $\mathbf{E}_{\text {tot }}$ & $\boldsymbol{a}[\AA \mathbf{\AA}]$ & $\boldsymbol{r}[\AA \mathbf{]}]$ & $\boldsymbol{a} / \mathbf{d}$ \\
\hline 0.55 & -2.5240 & 0.07 & 0.416 & 0.127 \\
\hline 0.60 & -2.8200 & 0.06 & 0.425 & 0.100 \\
\hline 0.65 & -3.0560 & 0.06 & 0.506 & 0.092 \\
\hline 0.70 & -3.2480 & 0.05 & 0.444 & 0.071 \\
\hline 0.746 & -3.3930 & 0.045 & 0.451 & 0.060 \\
\hline 0.80 & -3.5340 & 0.04 & 0.459 & 0.050 \\
\hline 0.85 & -3.6420 & 0.04 & 0.467 & 0.047 \\
\hline 0.9 & -3.7330 & 0.03 & 0.471 & 0.033 \\
\hline 1.00 & -3.8680 & 0.01 & 0.479 & 0.010 \\
\hline 1.10 & -3.9770 & 0.02 & 0.490 & 0.018 \\
\hline 1.20 & -4.0530 & 0.015 & 0.496 & 0.013 \\
\hline 1.40 & -4.1560 & 0.007 & 0.506 & 0.005 \\
\hline 1.60 & -4.2181 & 0.005 & 0.513 & 0.003 \\
\hline 2.00 & -4.2840 & 0.003 & 0.52 & 0.002 \\
\hline
\end{tabular}

designated as intranuclear-antipodal, intranuclear-sympodal, extranuclear-antipodal, and extranuclear-sympodal. Since in any case a variety of combinations of the relevant parameters exists fulfilling the given condition, the total energy has to be introduced for determining the preferred stable state which is given by an energy minimum. Thus it is necessary to additionally take into account this basic energy principle, which has been likewise applied in the classical theory for determining bond lengths, originally described by Heitler and London [12]. As a consequence, only those arrays come into question where for every bond length (i.e. distance $d$ ) an energy minimum exists, altogether delivering a distance/energy curve. Finally, the optimal distance is apparent when the total energy is minimal but lower than the total energy of the separated H-atoms. Further positions on this curve are also possible, admitting oscillations of the nuclei around the distance which corresponds to the minimal energy state. But these oscillations are not quantized because the initial quantum condition is maintained thereby. They are due to thermal motion and induced by the kinetic translation energy of the molecules which likewise is not quantized.

As the computations yielded, solely two of the four conceivable the H-atom according to Bohr's model. Thereby, the frequencies of the spin and of the revolution are identically equal which leads to a standing wave. However, in contrast to the classical quantum theory where the occurrence area of the electron is spherical even in the ground state, here the electron trajectory is planar and well defined. Solely in the excited states the trajectories get three dimensional - but still well-defined -, as has been shown in the previous treatise [6].

Applying the same concept for the $\mathrm{H}_{2}$-molecule, i.e. assuming the maintenance of the basic angular momentum for both $\mathrm{H}$-atoms at the $\mathrm{H}-\mathrm{H}$ bond, a comparatively simple computation method for the intrinsic variables could be found regarding several force equilibria. However, the relation could not be formulated generally but had to be adapted to the four conceivable arrays, arrays come into question, namely the intranuclear-antipodal and the extranuclear-sympodal one. But, as Figure 7 reveals, only the former one enables a stable state being given at a bond length of $0.746 \times 10^{-10} \mathrm{~m}$ (or $\AA$ ), and hence matching perfectly the desired value determined by XRD-crystallography. This result exceeds the accuracy of the next best of the diverse results found in the literature with respect to the statistical orbital theory based on Heitler's approach, namely $0.71 \AA ̊$ [14].

However, as Figure 8 reveals, the difference between the resulting bond energies for the two methods is enormous, being not explicable by slight computation defects. Referred to one mole hydrogen molecules, it is $-410 \mathrm{~kJ}$ in the case of the classical method, compared to $-168 \mathrm{~kJ}$ in the case of the here described own method. If these values are compared with the experimentally determined literature value of $-436 \mathrm{~kJ}$, the traditional method 


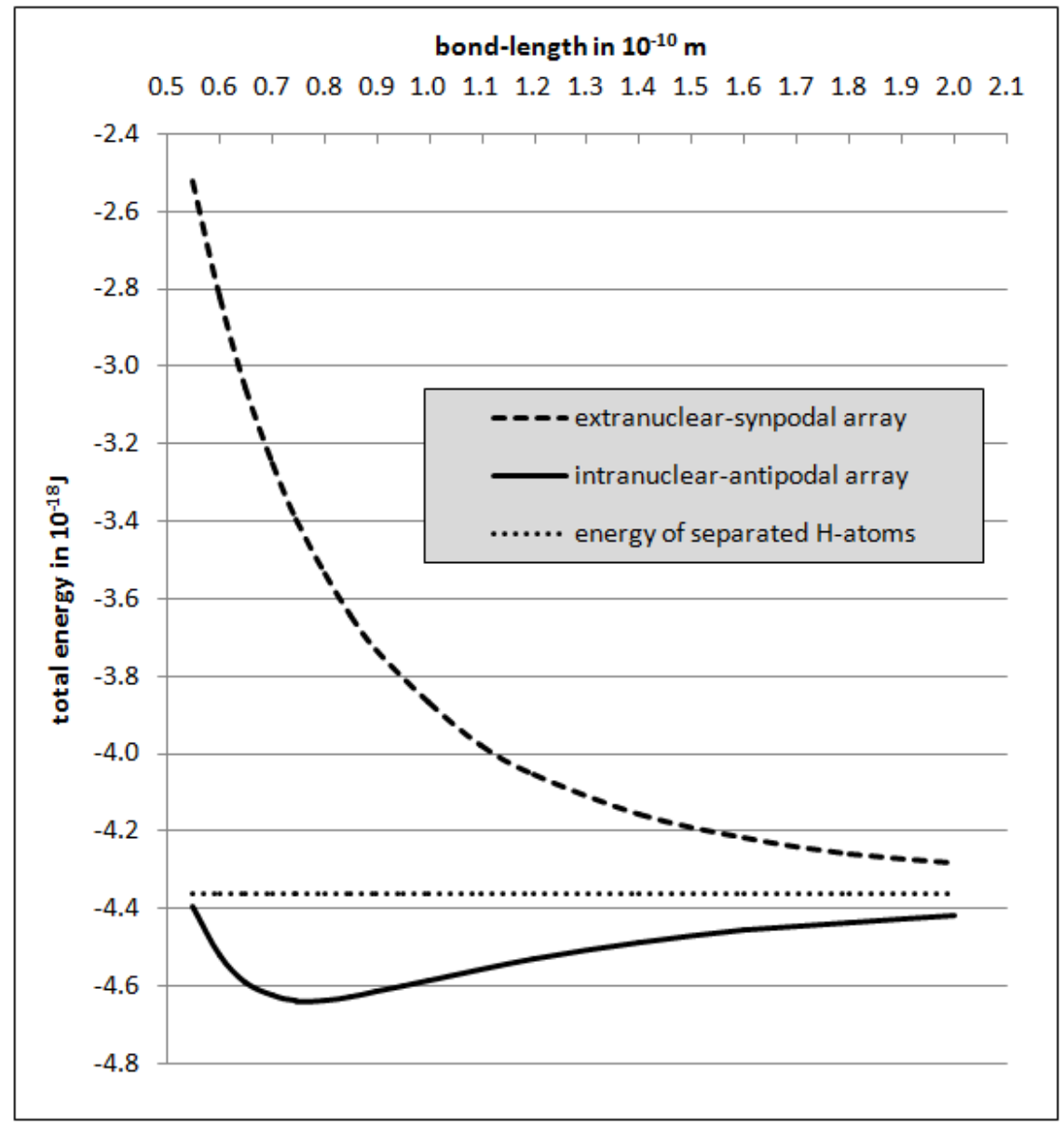

Figure 7: Bond-length/energy comparison of the two preferred arrays.

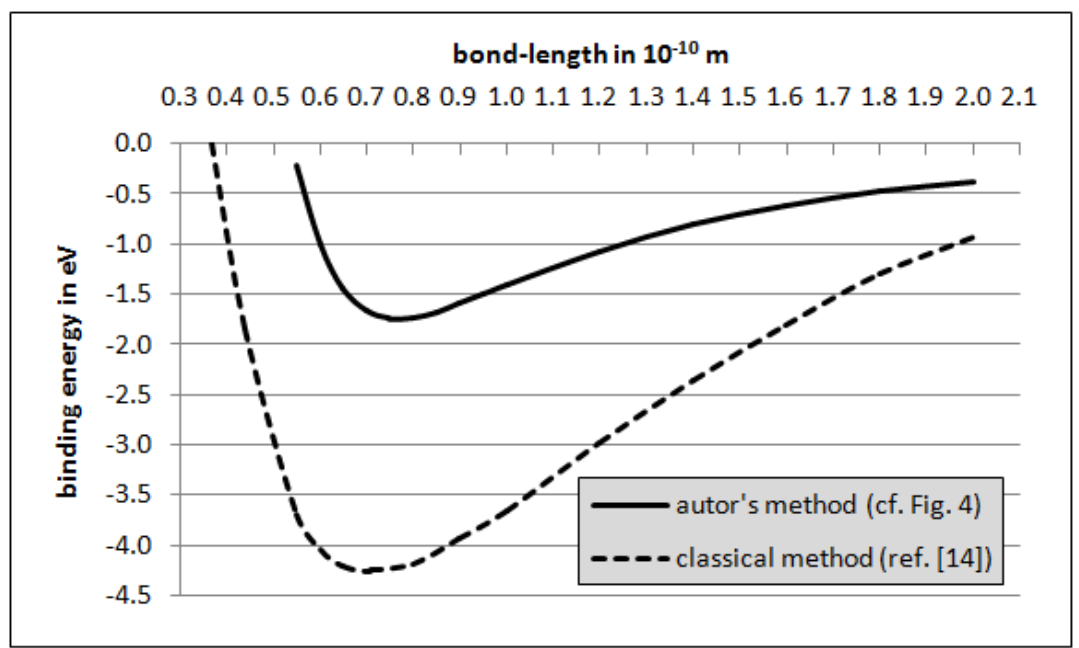

Figure 8: Bond-length/energy comparison between the results of the author's method (intranuclear-antipodal) and those of the classical Heitler-London method according to [14], the energy being converted into $\mathrm{eV}$ and related to the energy of the two separated $\mathrm{H}$-atoms. 
appears to be accurate. However, as a consideration of plausibility yielded, this literature value is probably not true but significantly too high, so it cannot be adduced to stringent evidence. But if the approach proposed here is correct, the existence of well-defined electron trajectories in $\mathrm{H}_{2}$ is proved by evidence, while the theory of Classical Quantum Mechanics cannot be maintained.

\section{Acknowledgement}

The present work has been carried out independently but not without the professional support of Dr Andreas Rüetschi (physicist, from the Swiss Federal Institute of Technology, Zurich).

\section{References}

1. Bohr N. On the Constitution of Atoms and Molecules. Phil. Mag. J. Sci. 1913;26:1-25.

2. Planck M. Ueber irreversible Strahlungsvorgänge. Ann. Phys. 1990;306(1):69-116. DOI: 10.1002/andp.19003060105.

3. Einstein A. Ueber einen die Erzeugung und Verwandlung des Lichts betreffenden heuristischen Gesichtspunkt. Ann. Phys. 1905;322(6):132-148. DOI: 10.1002/andp.19053220607.

4. Balmer JJ. Notiz über die Spectrallinien des Wasserstoffs. Ann. Phys. Chem. 1885;261(5):80-87. DOI:10.1002/andp.18852610506.

5. Mark $\mathrm{H}$ and Wierl R. Atomformfaktorbestimmung mit Elektronen. Zeitschrift für Physik. 1930;60(11-12):741-753.

6. Allmendinger T. A Classical Approach to the De Broglie-Wave Based on Bohr's H-Atom-Model. Int. Journal of Applied Mathematics and Theoretical Physics. 2016;2(1):1-15. DOI: 10.11648/j. ijamtp.20160201.11.
7. Uhlenbeck GE and Goudsmith S. Ersetzung der Hypothese vom unmechanischen Zwang durch eine Forderung bezüglich des inneren Verhaltens jedes einzelnen Elektrons. Naturwissenschaften. 1925;13(47):953.

8. Uhlenbeck GE and Goudsmith S. Spinning Electrons and the Structure of Spectra. Nature. 1926;117:264-265. DOI:10.1038/117264a0

9. Uhlenbeck GE and Goudsmith S. The Motion of the Spinning Electron. Nature. 1926;117:514.

10.Zeeman P. On the influence of Magnetism on the Nature of the Light emitted by a Substance. Phil. Mag. 1897;43(262):226-232.

11.Zeeman P. Doublets and triplets in the spectrum produced by external magnetic forces. Phil. Mag. 1897;44(268):55-59.

12. Heitler W and London F. Wechselwirkung neutraler Atome und homöopolare Bindung nach der Quantenmechanik. Zeitschrift für Physik. 1927;44(6-7):455-472.

13. Heitler W. Elementare Wellenmechanik. Friedr. Vieweg \& Sohn, Braunschweig. 1961.

14. Haken H and Wolf HC. Molekülphysik und Quantenchemie. Springer, $6^{\text {th }}$ edition, 2006.

15. Bichowsky FR and Copeland LC. The Heat of Formation of Molecular Hydrogen. J. Amer. Chem. Soc. 1928;50(5):1315-1322.

16. Knudsen M. Die Molekularströmung der Gase durch Öffnungen und die Effusion. Ann. Phys. 1909;333(5):999-1016. DOI: 10.1002/ andp.19093330505.

17. Chupka WA and Inghram MG. Direct Determination of the Heat of Sublimation of Carbon with the Mass Spectrometer. J. Phys. Chem. 1955;59(2):100-104. DOI: 10.1021/j150524a002.

18. Gingerich KA. Gaseous Metal Nitrides. IV. The Dissociation Energy of Cerium Mononitride. J. Chem. Phys. 1971;54(9):3720-3722. 